\title{
Elderly Patients With Single Brain Metastasis - Overall Survival After Surgery Plus Whole-Brain Irradiation and a Radiation Boost
}

\author{
DIRK RADES ${ }^{1}$, TRANG NGUYEN ${ }^{1}$ and STEVEN E. SCHILD ${ }^{2}$ \\ ${ }^{1}$ Department of Radiation Oncology, University of Lübeck, Lübeck, Germany; \\ ${ }^{2}$ Department of Radiation Oncology, Mayo Clinic, Scottsdale, AZ, U.S.A.
}

\begin{abstract}
Background/Aim: Elderly patients with a single brain metastasis likely benefit from personalized treatment protocols. To add to treatment personalization, a survival score was generated for these patients. Patients and Methods: This retrospective study included 36 elderly patients, each with a single brain metastasis, who received surgery followed by whole-brain irradiation and a radiation boost. Six pre-treatment characteristics were evaluated regarding survival, namely age, gender, Karnofsky performance score (KPS), type of primary tumor, noncerebral metastasis and interval from diagnosis of the neoplasm until surgery. Results: When applying the Cox regression model, $\operatorname{KPS}(p=0.005)$ and tumor type $(p=0.018)$ were significant and incorporated in the score. Based on 12month survival probabilities, three groups of 6-9 $(n=5), 10$ $11(n=15)$ and 14-19 points $(n=16)$ were formed, with 12month survival rates of $0 \%, 33 \%$ and $100 \%$, respectively $(p<0.0001)$. Conclusion: A survival score was generated specifically for elderly patients with a single brain metastasis that can improve personalization of their treatment.
\end{abstract}

Patients with a single brain metastasis are less common than patients with multiple lesions (1). If the brain lesion is surgically accessible and the patients are medically operable, they may be candidates for resection (1-4). Outcomes of patients with a single brain metastasis may be further improved with post-operative radiation therapy (5). A

This article is freely accessible online.

Correspondence to: Professor Dirk Rades, MD, Department of Radiation Oncology, University of Lubeck, Lubeck, Ratzeburger Allee 160, 23562 Lubeck, Germany. Tel: +49 45150045401, Fax: +49 45150045404, e-mail: rades.dirk@gmx.net

Key Words: Elderly patients, single brain metastasis, surgery, whole-brain irradiation, radiation boost, overall survival. previous study of patients aged $\geq 65$ years with one or two brain metastases compared four treatment protocols, namely whole-brain irradiation (WBI) alone, radiosurgery alone, surgery followed by WBI and surgery followed by WBI plus a radiation boost to the resection caves (6). The combined approach of surgery followed by WBI plus a boost achieved the best outcomes at 1 year in terms of significantly better survival, local control of the resected lesions and overall cerebral control. Thus, surgery followed by WBI plus a boost appeared preferable for elderly patients with one or two brain metastases. However, for many of these patients an invasive procedure such as brain surgery can be burdensome, particularly if they are impaired with underlying diseases. Moreover, in the previous study, the differences between the four treatments were less prominent at 6 months than at 1 year (6). Considering these results, an aggressive approach such as surgery followed by WBI plus a boost appeared more appropriate for longer-term survivors with comparably little co-morbidity. Therefore, it is important for the treating physicians to identify prior to the start of treatment a) patients who will likely live long enough to benefit from an aggressive multidisciplinary regimen and $b$ ) those patients who may not benefit. This study aimed to provide a score to support the estimation of a patient's survival prognosis and, thereby, contribute to the identification of patients who will likely live long enough to gain adequate benefit from such an aggressive regimen.

\section{Patients and Methods}

This retrospective study included 36 patients, each with a single brain metastasis, who were at least 65 years of age and had received surgery (resection of the metastasis) followed by WBI and a radiation boost to the metastatic site. The study was approved by the Ethics Committee of the University of Lubeck. The goal of this study was the development of a score that allowed the estimation of the 12-month survival. Six pre-treatment characteristics (Table I) were evaluated with respect to survival, including age ( $\leq 67 \mathrm{vs.} \geq 68$ years, median: 67 years), gender, Karnofsky performance score 
Table I. Patient, tumor and treatment characteristics.

\begin{tabular}{lc}
\hline Characteristic & Number of patients (\%) \\
\hline Age & $19(53)$ \\
$\leq 67$ Years & $17(47)$ \\
$\geq 68$ Years & \\
Gender & $11(31)$ \\
Female & $25(69)$ \\
Male & \\
KPS & $21(58)$ \\
$70-80$ & $15(42)$ \\
$90-100$ & \\
Type of primary tumor & $5(14)$ \\
Breast cancer & $14(39)$ \\
NSCLC & $7(19)$ \\
RCC/melanoma & $6(17)$ \\
GI-cancers & $4(11)$ \\
Other types & \\
Non-cerebral metastasis & $6(17)$ \\
Yes & $30(83)$ \\
No & \\
Interval from first diagnosis of neoplasm & \\
until surgery & $18(50)$ \\
$\leq 21$ Months & $18(50)$ \\
$\geq 22$ Months & \\
\hline
\end{tabular}

KPS: Karnofsky performance score; NSCLC: non-small cell lung cancer; RCC: renal-cell carcinoma; GI: gastrointestinal.

(KPS 70-80 vs. 90-100, median: 80), type of primary tumor [breast cancer $v s$. non-small cell lung cancer (NSCLC) vs. renal-cell carcinoma (RCC)/melanoma $v s$. gastrointestinal cancers $v s$. other tumor types], non-cerebral metastasis (yes $v s$. no) and interval from first diagnosis of the neoplasm until surgery of brain metastasis $(\leq 21$ $v s . \geq 22$ months, median: 21.5 months). Radiation therapy regimens included $10 \times 3$ Gy of WBI followed by a boost of $5 \times 3$ Gy $(n=16)$, $20 \times 2$ Gy of WBI followed by a boost of $5 \times 2$ Gy $(n=12), 10 \times 3 \mathrm{~Gy}$ of WBI followed by a boost of $3 \times 3$ Gy $(n=6), 10 \times 3$ Gy of WBI followed by a boost of $10 \times 2$ Gy $(n=1)$ and $14 \times 2.5$ Gy plus a simultaneous integrated boost of $7 \mathrm{~Gy}(n=1)$.

Time to death was referenced from the day of surgery. The characteristics that proved to be significantly $(p<0.05)$ associated with survival or showed a trend $(p<0.10)$ for such an association on univariate analysis (Kaplan-Meier method and log-rank test), were analyzed for independence using a Cox regression model. Characteristics achieving significance $(p<0.05)$ in the Cox regression analysis were incorporated in the newly developed score. Initially, scoring points for each characteristic were devised by dividing the 12 -month survival rates in percentage by 10 . Afterwards, these scoring points were added for each individual patient.

\section{Results}

Median follow up was 13.5 months (range $=1-66$ months) in all 36 patients and 16.5 months (range=7-66 months) in those 10 patients alive at their last contact.

The median survival time of all 36 patients was 17 months, and the 6- and 12-month survival rates were $75 \%$
Table II. Six- and 12-month survival rates of the evaluated characteristics.

\begin{tabular}{|c|c|c|c|}
\hline Characteristic & 6 months $(\%)$ & 12 months $(\%)$ & $p$-Value \\
\hline \multicolumn{4}{|l|}{ Age } \\
\hline$\leq 67$ Years & 79 & 62 & \multirow[t]{2}{*}{0.122} \\
\hline$\geq 68$ Years & 71 & 53 & \\
\hline \multicolumn{4}{|l|}{ Gender } \\
\hline Female & 73 & 62 & \multirow[t]{2}{*}{0.736} \\
\hline Male & 76 & 56 & \\
\hline \multicolumn{4}{|l|}{ KPS } \\
\hline $70-80$ & 67 & 38 & \multirow[t]{2}{*}{0.006} \\
\hline $90-100$ & 87 & 87 & \\
\hline \multicolumn{4}{|c|}{ Type of primary tumor } \\
\hline Breast cancer & 80 & 60 & \multirow[t]{5}{*}{0.061} \\
\hline NSCLC & 79 & 57 & \\
\hline RCC/melanoma & 100 & 100 & \\
\hline GI-cancers & 50 & 17 & \\
\hline Other types & 50 & 50 & \\
\hline \multicolumn{4}{|c|}{ Non-cerebral metastasis } \\
\hline Yes & 50 & 25 & \multirow[t]{2}{*}{0.086} \\
\hline No & 80 & 63 & \\
\hline \multicolumn{4}{|c|}{$\begin{array}{l}\text { Interval from first diagnosis } \\
\text { of neoplasm until surgery }\end{array}$} \\
\hline$\leq 21$ Months & 61 & 44 & \multirow[t]{2}{*}{0.273} \\
\hline$\geq 22$ Months & 89 & 71 & \\
\hline
\end{tabular}

KPS: Karnofsky performance score; NSCLC: non-small cell lung cancer; RCC: renal-cell carcinoma; GI: gastrointestinal; bold $p$-value is significant.

Table III. Independent predictors of 12-month survival and related scoring points.

\begin{tabular}{lcc}
\hline Characteristic & 12-month survival (\%) & Scoring points \\
\hline KPS & 38 & \\
$70-80$ & 87 & 4 \\
$90-100$ & & 9 \\
Type of primary tumor & 60 & 6 \\
Breast cancer & 57 & 6 \\
NSCLC & 100 & 10 \\
RCC/melanoma & 17 & 2 \\
GI-cancers & 50 & 5 \\
Other types & & \\
\hline
\end{tabular}

KPS: Karnofsky performance score; NSCLC: non-small cell lung cancer; RCC: renal-cell carcinoma; GI: gastrointestinal.

and $58 \%$. When the univariate analyses were performed (Table II), a KPS of 90-100 ( $p=0.006)$ was significantly associated with a more favorable survival. In addition, a trend was found for comparably favorable types of primary tumor, namely breast cancer, NSCLC and RCC/melanoma, $(p=0.061)$ and absence of non-cerebral metastasis $(p=0.086)$. When applying the Cox regression model, KPS of 90-100 [risk ratio $(\mathrm{RR})=3.44,95 \%$ confidence interval $(\mathrm{CI})=1.44$ - 


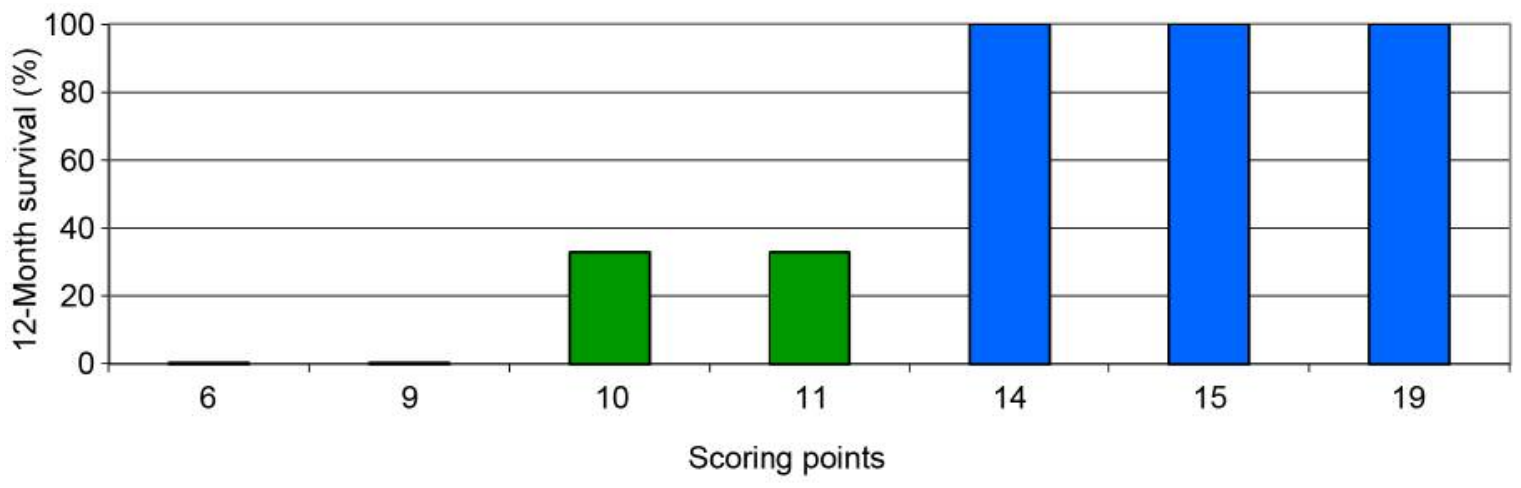

Figure 1. Survival rates of the different scoring points at 12 months following surgery.

9.09, $p=0.005$ ] and favourable types of primary tumor $(\mathrm{RR}=1.22,95 \% \mathrm{CI}=1.04-1.43, p=0.018)$ were significant, whereas absence of non-cerebral metastasis $(\mathrm{RR}=2.39$, 95\% CI=0.65-7.26, $p=0.176$ ) did not achieve significance. Thus, KPS and type of primary tumor were incorporated in the score as described above.

The scoring points of these two characteristics are shown in Table III. When applying this methodology, individual patients received scores that ranged between 6 and 19 points (Figure 1). Based on 12-month survival probabilities, three survival groups were formed: 6-9 points $(n=5), 10-11$ points $(n=15)$ and $14-19$ points $(n=16)$. The 12-month survival rates of these groups were $0 \%, 33 \%$ and $100 \%$, respectively (Figure 2, $p<0.0001$ ).

\section{Discussion}

Several treatment options exist for elderly patients with a single brain metastasis including WBI alone, radiosurgery alone and surgery followed by WBI with or without a radiation boost to the resection cavity $(1,6)$. A retrospective study of 164 patients with one or two brain metastases compared these treatments according to the survival, intracerebral control and local control they achieved (6). At 12 months, the highest survival rate was observed after surgery plus WBI and a boost, i.e. $61 \%$ compared to $40 \%$ after radiosurgery alone, $27 \%$ after surgery plus WBI and $13 \%$ after WBI alone $(p<0.001)$. Surgery plus WBI and a boost was also significantly superior to the other regimens with respect to 12 -month intracerebral control (79\% vs. 55\%, $36 \%$ and $17 \%$, respectively, $p<0.001$ ) and local control of the resected lesions ( $84 \%$ vs. $68 \%, 43 \%$ and 19\%, respectively, $p<0.001)$. At 6 months, the differences between the four regimens were less prominent (6). For example, the 6-month rates of survival, intracerebral control and local control were $78 \%, 82 \%$ and $86 \%$, respectively, after surgery plus WBI and a boost compared to $56 \%, 48 \%$ and $53 \%$, respectively, after

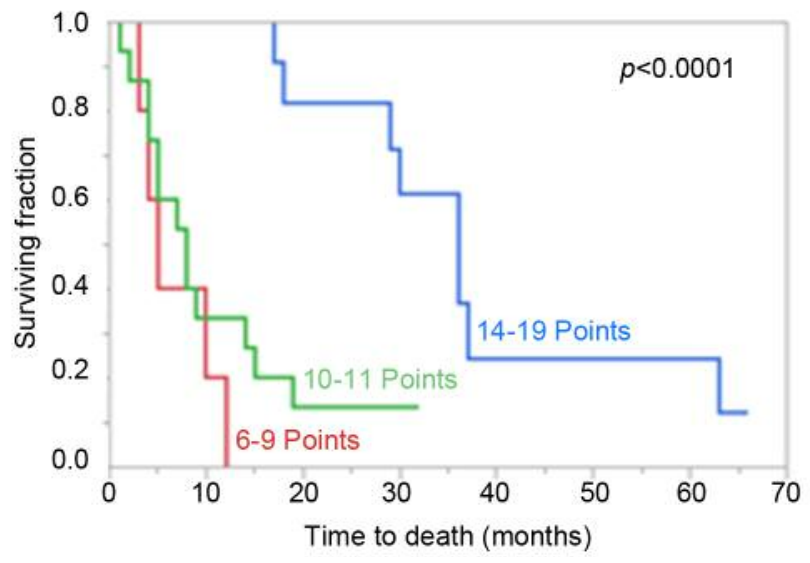

Figure 2. Kaplan-Meier curves of the patient-groups achieving 6-9 points $(n=5), 10-11$ points $(n=15)$ and 14-19 points $(n=16)$, respectively.

WBI alone. Thus, patients surviving for $\geq 12$ months had a greater benefit from the most aggressive approach (surgery followed by WBI and a radiation boost) than patients surviving for only 6 months. Since such an aggressive treatment program can be very stressful for elderly patients, the potential benefits should be carefully weighed against potential toxicities and complications. The benefit-risk assessment should also take into account a patient's survival prognosis, since benefit increases with lifetime. Therefore, it is important to accurately estimate a patient's remaining lifespan before considering an aggressive treatment protocol.

This study created a new survival score for elderly patients with a single brain metastasis, developed in a cohort treated with surgery plus WBI and a radiation boost. Based on the independent prognostic factors, KPS and type of primary tumor, we designed three groups with different 12-month survival probabilities. In the 6-9 points group, no patient survived as long as 12 months following surgery, and only $40 \%$ of these patients survived for 6 months. Therefore, these 
patients appeared less than ideal candidates for an aggressive treatment including neurosurgical resection (6). They may be better treated with radiosurgery alone that achieved the second-best results regarding survival, intracerebral control and local control in the previous study (6). Patients of the 1011 points group had 6- and 12-month survival rates of $60 \%$ and $33 \%$, respectively. Since the 12 -month survival rate was, although better than in the 6-9 points group, also not convincingly long, surgery plus WBI and a boost may be limited to selected patients. The majority of these patients may be considered for radiosurgery alone. When wishing to combine radiosurgery with novel targeted therapies, which is becoming increasingly popular for younger patients, one should be aware that these new systemic drugs can lead to severe adverse events particularly in elderly patients $(7,8)$.

In contrast to these two groups (5-9 points and 10-11 points), patients of the 14-19 points group had an excellent long-term survival with 12-month and 24-month survival rates of $100 \%$ and $82 \%$, respectively (Figure 2). These patients appear to benefit from surgery plus WBI and a radiation boost in terms of increased survival, intracerebral control and local control (6). They may also be considered for WBI plus radiosurgery, which provided similar 1-year outcomes as surgery plus WBI and a boost in a matched-pair study of 94 patients with one or two brain metastases and a matched-pair study of 92 patients of any age with a single lesion $(2,9)$. Studies comparing these two treatments in elderly patients alone are lacking.

The role of WBI in addition to a local therapy is currently under debate. Two older studies, a randomized trial $(n=95)$ and a retrospective study $(n=85)$ compared surgical resection alone to resection plus WBI for a single brain metastasis (3, 4). In the randomized trial, WBI led to increased 1-year local control $(90 \%$ vs. $54 \%, p<0.001)$ and intracerebral control ( $82 \%$ vs. $30 \%, p<0.001$ ) but not survival (median 11 months in both groups) (3). In the retrospective study, WBI resulted in increased 1-year local control $(79 \%$ vs. $15 \%, p<0.05)$ and survival (median 23 vs. 11.5 months, $p<0.05$ ) (4). More recently, two randomized trials demonstrated that WBI when added to radiosurgery resulted in a significantly higher rate of cognitive decline after a few months $(10,11)$. However, in both trials, significantly more intracerebral recurrences were found after 12 months in the radiosurgery alone arm. One has to be aware that intracerebral progression can also be associated with impairment of cognitive function, as well as the need for further therapy (12). Patients who have a considerable risk of developing an intracerebral recurrence after radiosurgery alone should be considered for upfront WBI (13). If a patient is assigned to a local therapy plus WBI, the risk of cognitive decline can be significantly reduced with WBI-techniques sparing the hippocampal areas and with administration of memantine, which is normally used for dementia and Alzheimer's disease (14-16). These options should be considered particularly for patients achieving 1419 points in our study, who are assigned to surgery plus WBI and a boost. When following the recommendations given in this article, the retrospective nature of the study with its risk of bias should be kept in mind.

In summary, a survival score was generated specifically for elderly patients with a single brain metastasis. This new score can improve personalization of their treatment by identifying patients who may be helped with an aggressive treatment protocol such as surgical resection followed by WBI and a radiation boost to the resection cavity.

\section{Conflicts of Interest}

On behalf of all Authors, the corresponding Author states that there are no conflicts of interest related to this study.

\section{Authors' Contributions}

D.R., T.N. and S.E.S, designed the study. T.N. and D.R. collected or provided data that were analyzed by D.R. and S.E.S. The draft of the article was written by D.R. and S.E.S. and subsequently reviewed and approved by all Authors.

\section{References}

1 Tsao MN, Rades D, Wirth A, Lo SS, Danielson BL, Gaspar LE, Sperduto PW, Vogelbaum MA, Radawski JD, Wang JZ, Gillin MT, Mohideen N, Hahn CA and Chang EL: Radiotherapeutic and surgical management for newly diagnosed brain metastasis(es): An American Society for Radiation Oncology evidence-based guideline. Pract Radiat Oncol 2: 210-225, 2012. PMID: 25925626. DOI: 10.1016/j.prro.2011.12.004

2 Rades D, Kueter JD, Meyners T, Pluemer A, Veninga T, Gliemroth $J$ and Schild SE: Single brain metastasis: resection followed by whole-brain irradiation and a boost to the metastatic site compared to whole-brain irradiation plus radiosurgery. Clin Neurol Neurosurg 114: 326-330, 2012. PMID: 22152784. DOI: 10.1016/j.clineuro.2011.10.042

3 Patchell RA, Tibbs PA, Regine WF, Dempsey RJ, Mohiuddin M, Kryscio RJ, Markesbery WR, Foon KA and Young B: Postoperative radiotherapy in the treatment of single metastases to the brain: a randomized trial. JAMA 280: 1485-1489, 1998. PMID: 9809728. DOI: 10.1001/jama.280.17.1485

4 Smalley SR, Schray MF, Laws ER Jr and O'Fallon JR: Adjuvant radiation therapy after surgical resection of solitary brain metastasis: association with pattern of failure and survival. Int $\mathrm{J}$ Radiat Oncol Biol Phys 13: 1611-1616, 1987. PMID: 3117742. DOI: 10.1016/0360-3016(87)90154-4

5 Rades D, Kueter JD, Gliemroth J, Veninga T, Pluemer A and Schild SE: Resection plus whole-brain irradiation versus resection plus whole-brain irradiation plus boost for the treatment of single brain metastasis. Strahlenther Onkol 188: 143-147, 2012. PMID: 22234538. DOI: 10.1007/s00066-011-0024-9

6 Rades D, Pluemer A, Veninga T and Schild SE: Comparison of different treatment approaches for one to two brain metastases in elderly patients. Strahlenther Onkol 184: 565-571, 2008. PMID: 19016014. DOI: 10.1007/s00066-008-1908-1 
7 Stera S, Balermpas P, Blanck O, Wolff R, Wurster S, Baumann R, Szüics M, Loutfi-Krauss B, Wilhelm ML, Seifert V, Rades D, Rödel C, Dunst J, Hildebrandt G, Arnold A, Meissner M and Kähler KC: Stereotactic radiosurgery combined with immune checkpoint inhibitors or kinase inhibitors for patients with multiple brain metastases of malignant melanoma. Melanoma Res 29: 187-195, 2019. PMID: 30802230. DOI: 10.1097/ CMR.0000000000000542

8 Petrelli F, De Stefani A, Trevisan F, Parati C, Inno A, Merelli B, Ghidini M, Bruschieri L, Vitali E, Cabiddu M, Borgonovo K, Ghilardi M, Barni S and Ghidini A: Combination of radiotherapy and immunotherapy for brain metastases: A systematic review and meta-analysis. Crit Rev Oncol Hematol 144: 102830, 2019. PMID: 31733443. DOI: 10.1016/j.critrevonc.2019.102830

9 Rades D, Kueter JD, Pluemer A, Veninga T and Schild SE: A matched-pair analysis comparing whole-brain radiotherapy plus stereotactic radiosurgery versus surgery plus whole-brain radiotherapy and a boost to the metastatic site for one or two brain metastases. Int J Radiat Oncol Biol Phys 73: 1077-1081, 2009. PMID: 18707817. DOI: 10.1016/j.ijrobp.2008.05.035

10 Chang EL, Wefel JS, Hess KR, Allen PK, Lang FF, Kornguth DG, Arbuckle RB, Swint JM, Shiu AS, Maor MH and Meyers CA: Neurocognition in patients with brain metastases treated with radiosurgery or radiosurgery plus whole-brain irradiation: a randomised controlled trial. Lancet Oncol 10: 1037-1044, 2009. PMID: 19801201. DOI: 10.1016/S1470-2045(09)70263-3

11 Brown PD, Jaeckle K, Ballman KV, Farace E, Cerhan JH, Anderson SK, Carrero XW, Barker FG 2nd, Deming R, Burri SH, Ménard C, Chung C, Stieber VW, Pollock BE, Galanis E, Buckner JC and Asher AL: Effect of radiosurgery alone vs. radiosurgery with whole brain radiation therapy on cognitive function in patients with 1 to 3 brain metastases: A randomized clinical trial. JAMA 316: 401-409, 2016. PMID: 27458945. DOI: $10.1001 /$ jama.2016.9839

12 Aoyama H, Tago M, Kato N, Toyoda T, Kenjyo M, Hirota S, Shioura H, Inomata T, Kunieda E, Hayakawa K, Nakagawa K, Kobashi G and Shirato H: Neurocognitive function of patients with brain metastasis who received either whole brain radiotherapy plus stereotactic radiosurgery or radiosurgery alone. Int J Radiat Oncol Biol Phys 68: 1388-1395, 2007. PMID: 17674975. DOI: $10.1016 /$ j.ijrobp.2007.03.048
13 Huttenlocher S, Dziggel L, Hornung D, Blanck O, Schild SE and Rades D: A new prognostic instrument to predict the probability of developing new cerebral metastases after radiosurgery alone. Radiat Oncol 9: 215, 2014. PMID: 25240823. DOI: 10.1186/ 1748-717X-9-215

14 Gondi V, Pugh SL, Tome WA, Caine C, Corn B, Kanner A, Rowley H, Kundapur V, DeNittis A, Greenspoon JN, Konski AA, Bauman GS, Shah S, Shi W, Wendland M, Kachnic L and Mehta MP: Preservation of memory with conformal avoidance of the hippocampal neural stem-cell compartment during wholebrain radiotherapy for brain metastases (RTOG 0933): a phase II multi-institutional trial. J Clin Oncol 32: 3810-3816, 2014. PMID: 25349290. DOI: 10.1200/JCO.2014.57.2909

15 Brown PD, Pugh S, Laack NN, Wefel JS, Khuntia D, Meyers C, Choucair A, Fox S, Suh JH, Roberge D, Kavadi V, Bentzen SM, Mehta MP and Watkins-Bruner D; Radiation Therapy Oncology Group (RTOG): Memantine for the prevention of cognitive dysfunction in patients receiving whole-brain radiotherapy: a randomized, double-blind, placebo-controlled trial. Neuro Oncol 15: 1429-1437, 2013. PMID: 23956241. DOI: 10.1093/neuonc/ not114

16 Brown PD, Gondi V, Pugh S, Tome WA, Wefel JS, Armstrong TS, Bovi JA, Robinson C, Konski A, Khuntia D, Grosshans D, Benzinger TLS, Bruner D, Gilbert MR, Roberge D, Kundapur V, Devisetty K, Shah S, Usuki K, Anderson BM, Stea B, Yoon H, Li J, Laack NN, Kruser TJ, Chmura SJ, Shi W, Deshmukh S, Mehta MP and Kachnic LA; for NRG Oncology: Hippocampal avoidance during whole-brain radiotherapy plus memantine for patients with brain metastases: Phase III trial NRG Oncology CC001. J Clin Oncol 38: JCO1902767, 2020. PMID: 32058845. DOI: $10.1200 / \mathrm{JCO} .19 .02767$
Received February 19, 2020

Revised March 25, 2020

Accepted March 26, 2020 